\title{
Regulation of Fibronectin and Laminin Binding Activity in Cultured Human Lymphoblastic Cell Lines
}

\author{
L.M. STOOLMAN,* T.-L. WANG, R. SITU, AND J. VARANI \\ Department of Pathology, University of Michigan Mcdical School, Ann Arbor, Michigan \\ 48109-0602
}

\begin{abstract}
The current study shows that a clonal derivative of the Jurkat cell line up-regulates both the avidity and density of the $\alpha 6 / \beta 1$ receptor in response to phorbol 12 myristate 13-acetate (PMA). This derivative attaches to fibronectin and, to a lesser degree, laminin constitutively. Adhesion and spreading are dramatically up-regulated following treatment with PMA. The response on fibronectin peaks within 4 hours, is insensitive to cyclohexaminde, can be blocked by monoclonal antibodies (Mabs) to the $\beta 1$ and $\alpha 5$ subunits of the $\beta 1$ family of integrins, and is not associated with increased expression of the $\alpha 5$ or $\beta 1$ epitopes at the cell surface. In contrast, the response on laminin is biphasic. The early phase parallels the responsc on fibronectin. The second phase peaks after 48-72 hours of treatment with PMA, is sensitive to cycloheximide, can be blocked by Mabs to the $\beta 1$ and $\alpha 6$ subunits, and is associated with increased expression of the $\alpha 6$ epitope. Both the density independent and dependent responses to PMA in Jurkat cells are blocked by the protein kinase inhibitor staurosporine. The HSB-2, CFM, Molt-4, and HPB-ALL T-lymphoblastic cell lines also up-regulate attachment to fibronectin and laminin following treatment with PMA. All four lines constitutively attach to fibronectin and show rapid up-regulation of attachment following treatment with PMA. None of the lines attach to laminin prior to PMA treatment; however, specific adhesion developed after 4-120 hours of treatment. The most mature lines (Jurkat and HPB-ALL) up-regulated adhesion on laminin more rapidly than the less phenotypically mature lines (CEM, Molt-4, and HSB -2). In summary, clonal derivatives of the Jurkat cell line up-regulated attachment to laminin through protein kinase dependent increases in $\alpha 6 / \beta 1$ receptor avidity and density. In addition, the expression of functional receptors for laminin is linked to developmental maturity in a series of T-lymphoblastic cell lines. (c) 1993 Wiley-Liss, Inc.
\end{abstract}

The accumulation of normal and malignant lymphoid cells in tissues in a multistep process. Adhesive interactions involving components of the extracellular matrix may contribute to several steps. In the quail, T-cell progenitors moving into the embryonic thymus express functional fibronectin receptors, follow a migratory route containing substantial amounts of fibronectin, and accumulate in laminin-rich areas of the thymic rudiment (Savagner et al., 1986; 1988). In the human, cross-linking of the T-cell receptor augments binding to both fibronectin and laminin (Shimizu et al., $1990 \mathrm{c})$. Furthermore, the interaction of lymphocytic integrins with fibronectin and laminin enhances proliferation in response to T-cell receptor cross-linking (Shimizu et al., 1990a). Thus the interaction of $\mathrm{T}$ cells with components of the extracellular matrix may foster the migration, retention, and proliferation of lymphoid cells in tissues.

Previous studies revealed marked differences in the expression of laminin and fibronectin binding activities on human T cells. Normal human thymocytes (Cardarelli and Pierschbacher, 1986, 1987) and a variety of T-lymphoblastic cell lines (Cardarelli et al., 1988;
Wilkins et al., 1991) showed constitutive or phorbol ester-induced binding to fibronectin but not to laminin. In keeping with these observations, $\beta 1$ integrins with fibronectin binding activity $(\alpha 1-5 / \beta 1)$ are commonly expressed on malignant lymphoid lines, whereas the major laminin binding receptor $\alpha 6 / \beta 1$ has not been reported (Wayner et al., 1988; Hemler et al., 1987; Takada et al., 1987; Wayner et al., 1989). In contrast, circulating normal lymphocytes constitutively express both fibronectin $(\alpha 3-\alpha 5 / \beta 1)$ and laminin $(\alpha 6 / \beta 1)$ receptors (Sanchez-Madrid et al., 1986; Shimizu et al., $1990 \mathrm{~b})$. The densities of the $\alpha 5$ and $\alpha 6 / \beta 1$ receptors increase further during conversion of naive into mem-

\footnotetext{
Received June 28, 1991; accepted October 8, 1992.
}

"To whom reprint requests/correspondence should be addressed.

Dr. Tai-Ling Wang is now at the Department of Pathology, ChinaJapan Friendship Hospital, Yinghua East Road, Hepingli, Choyang District 100013, Beijing, PRC.

Dr. Rui Situ is now at the Department of Pathology, Jinan Medical College, GuangZhow, PRC. 
ory cells (Shimizu et al., 1990c). These independent observations suggest that expression of the $\alpha 6 / \beta 1$ receptor and laminin binding activity are developmentally regulated in both normal and malignant $\mathrm{T}$ lymphocytes.

The current study uses a clonal variant of the Jurkat cell line to examine the function and regulation of $\alpha 5 / \beta 1$ and $\alpha 6 / \beta 1$. In addition, evidence is presented supporting a link between developmental stage and expression of functional receptors for fibronectin and laminin in T-lymphoblastic cell lines.

\section{MATERIALS AND METHODS Cells}

The Jurkat variant used for most of the studies (J11) was isolated via limited dilution cloning from a strain of Jurkat cells described previously (Stoolman and Ebling, 1989). The parent line and all derivatives express markers consistent with late thymic differentiation including CD3, CD4 (weak), and CD2. The J11 variant expresses the peripheral lymph node homing receptor L-selectin (Leu 8, TQ1, LAM-1, DREG, and LECAM-1) at a higher level than the parent line and resting peripheral blood lymphocytes.

The phenotypes of the HSB-2, CEM, Molt-4, and HPB-ALL T-lymphoblastic cell lines have been extensively reported in the literature (Delia et al., 1982; Carrel et al., 1987; Stoolman and Ebling, 1989). In brief, the HSB-2 line expresses pan-T antigens exclusively, thus resembles normal thymic progenitors. The CEM and Molt-4 lines express CD1, CD4, and CD8, mimicking the features of stage II (common) thymocytes. HBPALL, like Jurkat, expresses complete T-cell receptor complexes (CD3 and WT31 epitopes) in addition to various pan- $\mathrm{T}$ antigens. This is consistent with features of stage II-III (mature) thymocytes. However, unlike Jurkat, HPB-ALL does not express the peripheral lymph node homing receptor. This molecule is normally expressed just prior to release of stage III thymocytes into the circulation and is present on $50-80 \%$ of circulating $\mathrm{T}$ and $\mathrm{B}$ lymphocytes.

\section{Chemicals}

Purified fibronectin and laminin were purchased from Collaborative Research (Boston, MA). Bovine serum albumin, fraction $\mathrm{V}$ (BSA), was obtained from Sigma Chemical Co. (St. Louis, MO). RPMI 1640, glutamine, and antibiotics for tissue culture were products of Gibco (Grand Island, NY). The monoclonal antibodies (Mabs) to the $\beta 1$ integrins were generous gifts of Dr. Caroline Damsky (AIIB2, B1E5, and J1B5) (Brown et al., 1989; Hall et al., 1990) and Dr. A. Sonnenberg (GoH3) (Sonnenberg et al., 1988). The specificities and blocking activities of these antibodies have been described previously. An additional monoclonal antibody specific for the $\alpha 4$ (clone HP2/1) chain was purchased from Amac (Portland, ME). Phorbol myristate acetate (PMA) was obtained from Sigma. Staurosporine was purchased from Kamiya Biochemical Corporation (Thousand Oaks, CA).

\section{Cell culture and treatments}

Cell lines were maintained in bicarbonate-buffered RPMI 1640 medium supplemented with $10 \%$ fetal bo- vine serum, penicillin-G, streptomycin, and fungizone at $2-15 \times 10^{5}$ cells/ml. Unless otherwise specified, treatment with PMA was initiated by adding $<1 \mathrm{ml}$ of freshly diluted, filter sterilized PMA to cells at $2-4 \times 10^{5}$ cells $/ \mathrm{ml}$ in growth medium. PMA stock solutions $(500 \mu \mathrm{M}$ in $100 \% \mathrm{DMSO})$ were stored at $-20^{\circ} \mathrm{C}$ for $<1$ month prior to use. The final concentration of PMA was $5 \mathrm{nM}$. DMSO alone at $0.01 \%$ had no effect on cell growth, differentiation, or extracellular matrix binding activity. Prolonged incubation with PMA resulted in growth arrest and cytotoxicity; therefore, dead cells were removed via sedimentation through ficoll prior to use in the binding assay if trypan blue uptake exceeded $15 \%$.

\section{Adhesion assays}

Fibronectin, laminin or BSA were diluted in RPMI 1640 medium without serum, added to 24 -well tissue culture plates $(300-500 \mu \mathrm{l}$ containing $1-25 \mu \mathrm{g} / \mathrm{well})$ and incubated for 2 hours at $37^{\circ} \mathrm{C}$ under $\mathrm{CO}^{2}$. After washing the plates with RPMI 1640 medium supplemented with $200 \mu \mathrm{g} / \mathrm{ml} \mathrm{BSA}$ (subsequently referred to as assay buffer), cells were added to triplicate wells (1 $\mathrm{ml}$ of assay buffer containing $5 \times 10^{5}$ cells) and incubated at $37^{\circ} \mathrm{C}$ for 4 hours. At the conclusion of the incubation, unattached cells were removed by first decanting the excess suspension and then gently adding $1 \mathrm{ml}$ of phosphate-buffered saline (PBS) down the walls of each well, rocking, decanting, and drying on absorbant paper. The cells remaining attached were counted using a Coulter ZX cell counter after quantitative removal with trypsin-EDTA.

\section{Surface marker analysis}

Indirect immunofluorescence assays for T-cell antigens were conducted in microtiter plates as follows. Antigen-specific mouse antihuman or rat antihuman (AIIB2, GoH3) monoclonal antibodies $(50 \mu 1)$ were combined with cells $\left(2.5 \times 10^{5}\right.$ in $\left.50 \mu \mathrm{l}\right)$ and incubated at $4^{\circ} \mathrm{C}$ for 30 minutes in PBS supplemented with $1 \%$ fetal bovine serum and $0.05 \%$ sodium azide (subsequently referred to as FA buffer). Cells were washed twice in FA buffer $(200 \mu \mathrm{l})$, resuspended, and combined with 50 $\mu \mathrm{l}$ of FITC-labeled goat antimouse IgG ( $\mathrm{Fab}^{\prime} 2$ fragments, Cappel Laboratories, West Chester, PA) for an additional 30 minutes $4^{\circ} \mathrm{C}$. After three washes in FA buffer, pellets were resuspended and combined with $200 \mu \mathrm{l}$ of $2 \%$ paraformaldehyde prior to cytometric analysis. Both primary and secondary antibodies were used at saturating concentrations.

In the experiment in Table 3, mean linear fluorescence (MLF) for each marker was determined in 5,000 cell samples using a Coulter Epics V Cytometer (FACS) as previously described (Stoolman and Ebling, 1989). The signals from all cells (positives and negatives) were included in the determinations so that changes in both the percentage of cells expressing a given marker and antigen density were reflected in the sample mean. The MLFs for each marker were normalized to that for nonspecific fluorescence, which was arbitrarily set to 1 . A 25-fold excess of pooled mouse IgG (control for T-cell markers) was used in the primary incubation to quantitate non-specific fluorescence. 


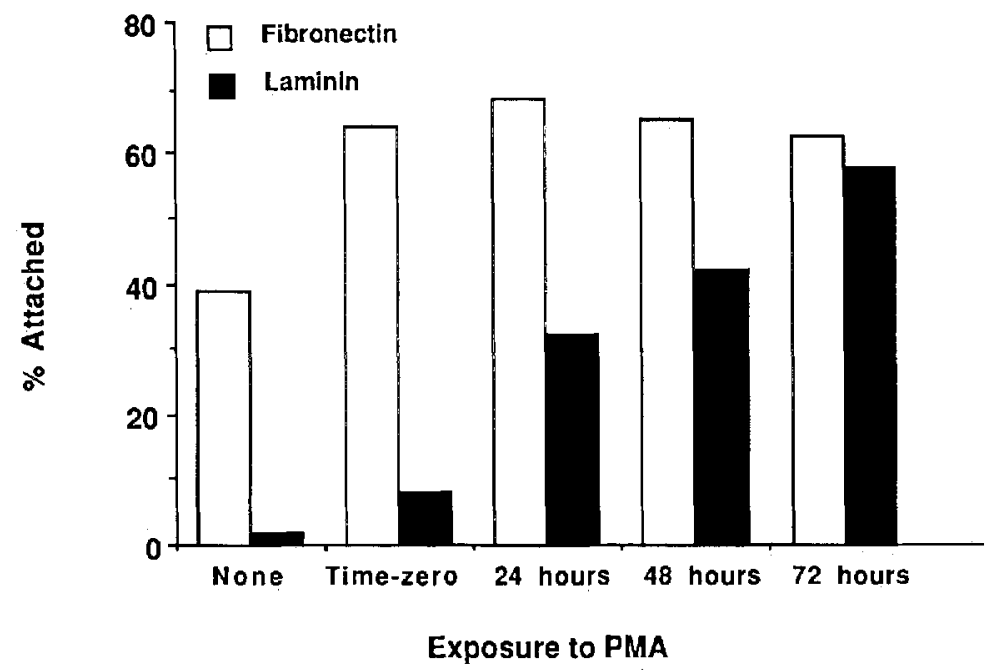

Fig. 1. Time-course for PMA-induced attachment of Jurkat cells to fibronectin and laminin. Adhesion assays were carried out as described in Materials and Methods. The incubation times reflect the duration of co-culture with PMA prior to the start of the adhesion assay. The adhesion assay includes a 4-hour incubation on substrate-coated plastic. Therefore, the "time zero" point reflects changes occurring during the first 4 hours of incubation.

\section{RESULTS \\ Characterization of Jurkat cell adhesion to fibronectin and laminin}

Jurkat cells from several sources (strains) were examined for constitutive and PMA-induced attachment to fibronectin and laminin. All Jurkat strains showed qualitative similarities in their patterns of attachment; however, there were quantitative differences. The J11 variant, derived by limited dilution cloning of the strain showing the highest adhesive activity, was used primarily.

Jurkat cells attached constitutively to fibronectin (Fig. 1). Treatment with PMA up-regulated attachment $\sim$ twofold and resulted in spreading of $>70 \%$ of the attached cells (Fig. 2) within 4 hours. Experiments using shorter periods of incubation in the attachment assay showed up-regulation within 1 hour in keeping with previous studies (Wilkins et al., 1991). In most cases, no further increases occurred with PMA treatment for as long as 120 hours. The level of constitutive attachment to laminin varied in different experiments; however, it was consistently below that observed on fibronectin. In contrast to fibronectin, PMA-treatment increased adhesion and spreading on laminin at both early and late time points. Adhesion peaked at 10-20fold over baseline after 48-72 hours of treatment. Polyclonal antisera to fibronectin and laminin were used to assess the the specificity of the adhesion response on both substrates. Figure 3 shows that antifibronectin inhibited Jurkat cell attachment to fibronectin but had no affect on adhesion to laminin. Conversely, antilaminin completely inhibited attachment to laminin but had no effect on attachment to fibronectin (Fig. 3).

We next examined the effects of cyclohexamide on Jurkat cell attachment to fibronectin and laminin. For these experiments, cells were pretreated for 48 hours with PMA in the absence or presence of cyclohexamide $\left(1 \times 10^{-7}\right.$ and $\left.5 \times 10^{-7} \mathrm{M}\right)$. In addition, cyclohexamide was added to Jurkat cells, with and without PMA, at the start of the adhesion assay. At these concentrations, cyclohexamide had no effect on basal or stimulated attachment on fibronectin (Fig. 4). In contrast, $5 \times 10^{-7}$ cyclohexamide significantly inhibited the attachment of PMA-treated Jurkat cells to laminin at 48 hours. Higher concentrations of cyclohexamide $\left(1 \times 10^{-6}\right.$ and $\left.5 \times 10^{-6} \mathrm{M}\right)$ were toxic to Jurkat cells after $24-48$ hours and therefore could not be used.

\section{Fibronectin and laminin receptors on Jurkat cells}

The next series of experiments evaluated the roles of selected $\beta 1$ integrins in the attachment of the J11 variant to fibronectin and laminin. Epitopes on the $\beta 1, \alpha 4$, and $\alpha 5$ chains were strongly expressed on untreated cells (Fig. 5). In contast, the $\alpha 6$ epitopes recognized by Mabs GoH3 (Fig. 5) and J1B5 (not shown) were expressed at significantly lower levels. Continuous treatment with PMA increased both the mean density and the percentage of cells expressing all but the $\alpha 4$ epitope. The $\alpha 6$ epitope showed the greatest increase over baseline expression, rising fourfold after 24 hours and up to sixfold after 48 hours (Table 1). Addition of cyclohexamide blocked these increases at the 24 hour time point (Fig. 5). Thus at least two known receptors for fibronec$\operatorname{tin}(\alpha 4 / \beta 1$ and $\alpha 5 / \beta 1)$ and one receptor for laminin $(\alpha 6 /$ $\beta 1$ ) were candidates for matrix adhesion receptors on the J11 variant.

The experiment depicted in Table 2 examined the inhibitory potency of several monoclonal antibodies to the $\beta 1$ integrins. The $\beta 1$-specific Mab AIIB 2 completely inhibited attachment of PMA-treated Jurkat cells to both fibronectin and laminin. The $\alpha 5$-specific Mab $\mathrm{B} 1 \mathrm{E} 5$ reduced attachment to fibronectin by $76 \%$ but did not significantly inhibit attachment to laminin. In contrast, the $\alpha 6$-specific Mab GoH3 blocked adhesion to laminin by $80 \%$ but had no affect on attachment to fibronectin. 

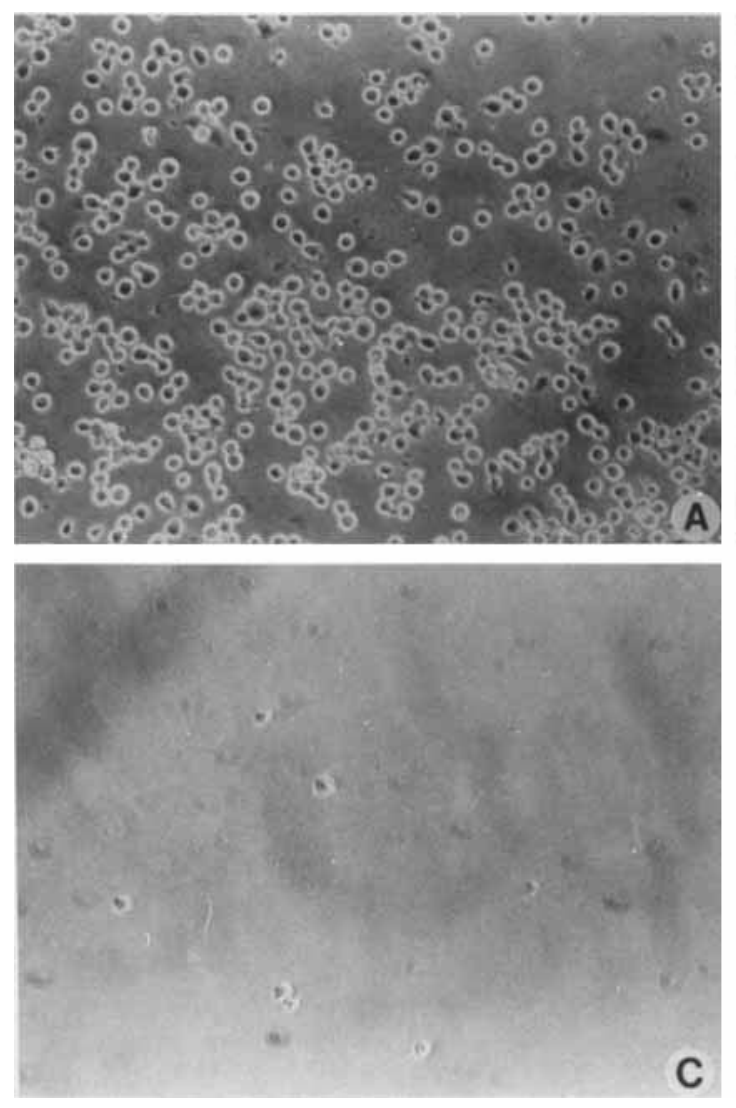

C

Fig. 2. Phase-contrast photomicrographs of Jurkat cells attached to fibronectin or laminin. A. Untreated Jurkat cells attached to fibronectin. B. Jurkat cells treated with PMA at the time of assay attached to fibronectin. C. Untreated Jurkat cells attached to laminin. D. Jurkat cells pre-treated with PMA for 2 days attached to laminin. In all cases,

\section{Effect of staurosporine on PMA-induced expression and function of matrix receptors in Jurkat cells}

Preincubation of Jurkat cells with the broad-spectrum protein kinase inhibitor staurosporine blocked PMA-induced increases in matrix receptor function and expression (Table 3 ). The combination of staurosporine and PMA increased trypan uptake by $\sim 10 \%$ relative to untreated cells and cells incubated with staurosporine alone. Therefore, the reduction in basal binding may reflect mild nonspecific toxicity. However, the reduction of PMA-dependent adhesion at both the 2- and 20-24-hour time points was far greater than that resulting from staurosporine alone. After 2 hours of incubation, staurosporine alone reduced the number of cells bound to laminin from $3 \%$ to $2 \%$ and to fibronectin from $32 \%$ to $27 \%$. In contrast, binding of PMA-treated cells was reduced from $13 \%$ to $1 \%$ and from $50 \%$ to $29 \%$, respectively. After 20 hours of incubation, staurosporine alone reduced the number of cells bound to laminin from $2 \%$ to $1 \%$ and to fibronectin from $29 \%$ to $22 \%$. Attachment of PMA-treated cells was reduced from $25 \%$ to $3 \%$ and $52 \%$ to $27 \%$, respectively. The combination of staurosporine and PMA had no effect on the levels of either the $\alpha 5$ or $\alpha 6$ epitopes at the 2 -hour time
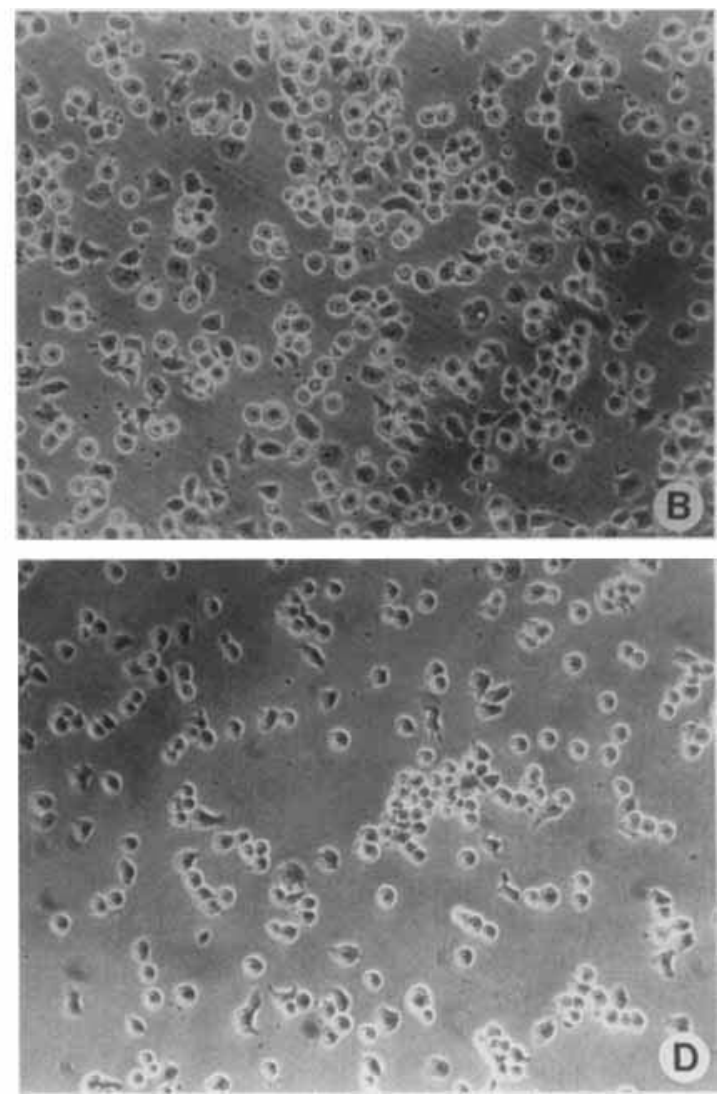

the cells were added to the substrates at time-zero and incubated for 4 hours. Following this, the nonattached cells were removed and the remaining cells were washed two times in assay buffer. They were then fixed in $2 \%$ glutaraldehyde and photographed under phase-contrast microscopy at $66 \times$ magnification.

point. However, the usual threefold increase in $\alpha 6$ expression at the 20 -hour time-point was reduced by $\sim 85 \%$.

\section{Attachment of other T-lymphoblastic lines to fibronectin and laminin}

Four additional T-lymphoblastic lines were examined for attachment to laminin and fibronectin. These were HSB-2, which resembles normal thymic progenitor cells, CEM and Molt-4, which mimic stage II (common) thymocytes and HPB-ALL, which like Jurkat, resemble cells late in thymic development. Pooled data from several experiments are shown in Table 4. All of the cell lines adhered to fibronectin, regardless of their stage of development. PMA treatment resulted in an approximate twofold increase in attachment within 4-8 hours, which did not increase further at later time points. In contrast, none of the lines showed significant constitutive attachment to laminin. PMA treatment resulted in attachment to laminin within 4-120 hours. The magnitude of the increase and the time course for its development differed amongst the cell lines. Jurkat and HPB-ALL showed increases at the earliest timepoint (Tables 3 and 4 ) with maximal levels achieved within 48-72 hours. In contrast, the Molt-4 and CEM 




Fig 3. Effects of rabbit polyclonal antibodies to fibronectin and laminin on Jurkat cell attachment to these matrix components. The substrates were prepared as described in Materials and Methods. Both substrates were then treated for 30 minutes at room temperature with a 1:10 dilution of antifibronectin serum, antilaminin serum, or normal rabbit serum. At the end of the incubation period, the sera were removed and the substrates washed in assay buffer. PMA-treated (48 hrs) Jurkat cells were then added. The percentage of attached cells after $\mathbf{4}$ hours of incubation was measured as described in Materials and Methods. Data points represent means and standard deviations based on triplicate samples per data point in a single experiment. The experiment was repeated two times with similar results.

lines did not show an increase until the 48-hour time point, with maximal levels achieved after 120 hours of incubation. The HSB-2 line displayed only a minimal increase after 5 days of continuous treatment.

\section{DISCUSSION}

In normal T-cells (Shimizu et al., 1990c), $\beta 1$ integrinmediated binding to laminin and fibronectin increases rapidly in response to treatment with phorbol esters and mitogenic Mabs. This initial response does not require new receptor synthesis, thus presumably reflects conversion of pre-existing receptors to a high avidity state. In addition, the density of multiple $\beta 1$-integrins is significantly higher on memory than on naive cells presumably reflecting induction of new receptor synthesis during antigen driven differentiation (Shimizu et al., 1990c). Thus normal T-cells regulate both the avidity and the density of the $\beta 1$ integrins in response to activation. Previous studies indicated that like normal cells, the Jurkat line responds to PMA with rapid, density independent upregulation of $\beta 1$-integrin function (Chan et al., 1991). However, induction of new receptor synthesis has not been reported in cell lines. The current study describes a variant of the Jurkat cell line in which PMA treatment increases the avidity, synthesis and surface expression of several $\beta 1$-integrins.

As reported previously for Jurkat cells, this variant binds constitutively to fibronectin and, to a lesser degree, laminin (Chan et al., 1991). PMA treatment re-

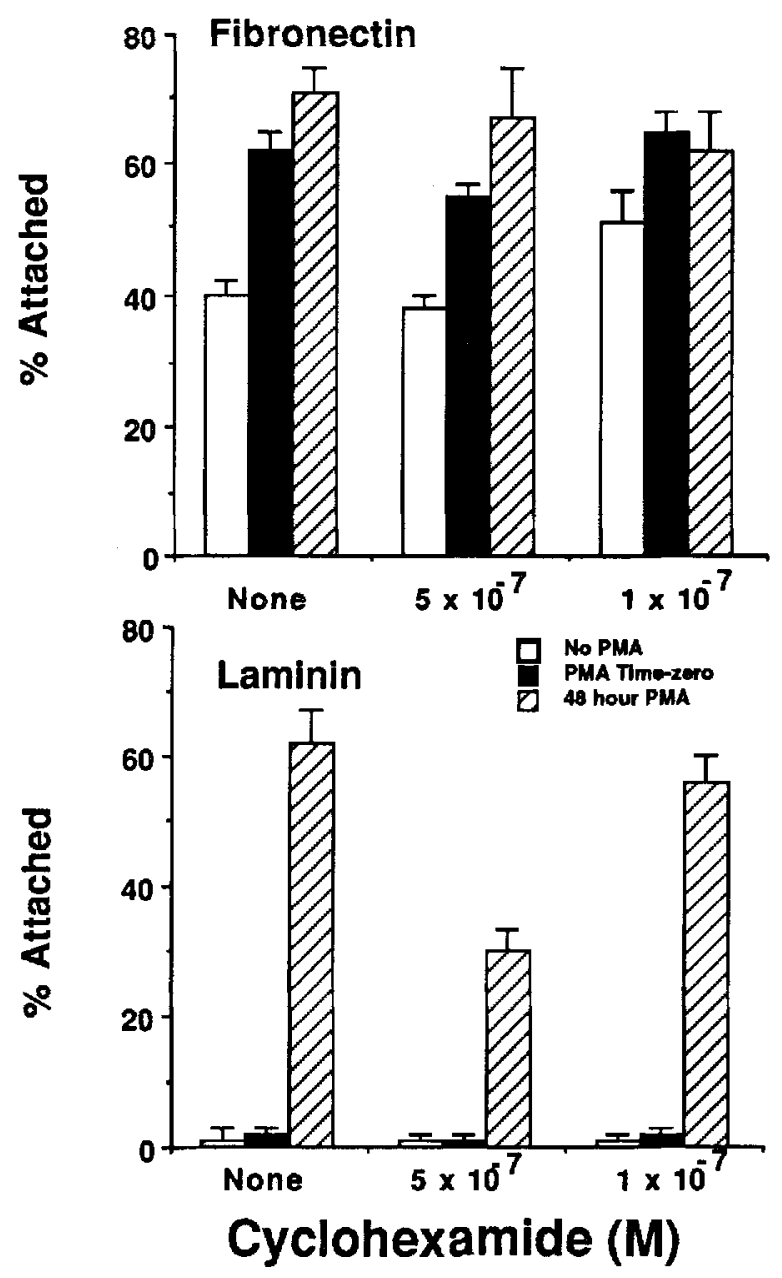

Fig. 4. Effects of cyclohexamide on PMA-induced up-regulation of Jurkat cell adhesion to fibronectin or laminin. Untreated Jurkat cells, Jurkat cells treated with PMA at the time of assay, and Jurkat cells pre-treated with PMA for 48 hours in the presence or absence of cyclohexamide were examined for attachment to fibronectin and laminin in the normal manner. Data points represent means and standard deviations based on triplicate samples in a single experiment. The difference in adhesion to laminin after treatment with PMA alone and PMA combined with $5 \times 10^{-7} \mathrm{M}$ cyclohexamide were statistically significant using the Student $t$-test $(P<0.05)$. The experiment was repeated four times with similar results.

sults in biphasic up-regulation of attachment. Marked spreading and a 2-5-fold increase in attachment to both matrix components develops within hours. This phase is not associated with an increase in receptor expression nor is it blocked by cyclohexamide; therefore, a change in receptor avidity is the most likely mechanism. Attachment to fibronectin peaks during this initial phase. However, adhesion and spreading on laminin continues to rise peaking at 10-20-fold over baseline after 48-72 hours of incubation. A sixfold increase in expression of the $\alpha 6$ epitope accompanies this second phase. Interestingly, the density of the $\beta 1$ epitope does not increase significantly suggesting that changes in alpha chain synthesis determine expression of the heterodimer at the cell surface. Inhibition of protein synthesis inhibits the late increases in both lami- 

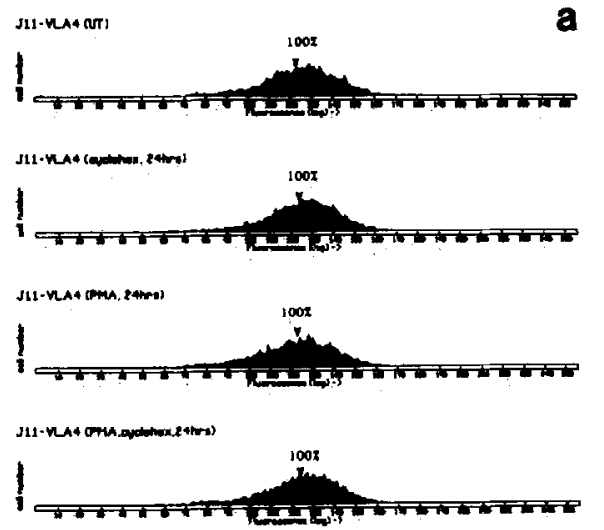

J11-XnN IN1

C





14



Fig. 5. Flow cytometric analysis of $\beta 1$ integrin expression on Jurkat cells. Cells were treated for 24 hours with $5 \times 10^{7} \mathrm{M}$ cyclohexamide (cyclohex, 24 hrs), 5 nM PMA (PMA, 24 hrs), or both cyclohexamide and PMA (PMA, cyclohex, $24 \mathrm{hrs}$ ). After washing, untreated (UT) cells and the three treated populations were stained for $\alpha 4$ (a), $\alpha 5$ (b), $\alpha 6$ (c), and $\beta 1$ (d) epitopes. Staining and flow cytometric analysis were carried out as described in Materials and Methods. Graphs show cell

TABLE 1. Up-regulation of $\beta 1$ integrins on the surface of PMA-treated Jurkat cells ${ }^{1}$

\begin{tabular}{lcccc}
\hline \multirow{2}{*}{$\begin{array}{l}\text { Monoclonal } \\
\text { antibody }\end{array}$} & Specificity & 4 Relative fluorescence intensity \\
\cline { 3 - 5 } & $\alpha 5$ & $133 \pm 20$ & $171 \pm 18$ & $241 \pm 13$ \\
B1E5 & $\alpha 6$ & $93 \pm 7$ & $480 \pm 40$ & $601 \pm 71$ \\
J1B5 & $\alpha 6$ &
\end{tabular}

${ }^{1}$ Flow cytometric indirect immunofluorescence studies were conducted as described in Materials and Methods. The mean fluoreseence intensities for ench marker (linear scale) were normalized to means obtained with untreated cells. Thus a value of 100 indicates that there has been no change in marker expression in the PMA-treated cells relative to untreated control cells. A value of 1.000 indicates a ten-fold increase. Data points represent means = ranges based on duplicate samples in single experiment. The experiment was repeated three times with similar results.

nin binding activity and the density of the $\alpha 6$ epitope. Thus new receptor synthesis contributes to the increase in laminin binding activity at late time points. The fact that attachment increases to a greater degree than receptor expression suggests the newly synthesized receptors are in a "high avidity" state. Therefore, maximal binding activity is a function of both receptor density and avidity in lymphoid cells. PMA augments adhesion through both mechanisms in Jurkat cells.
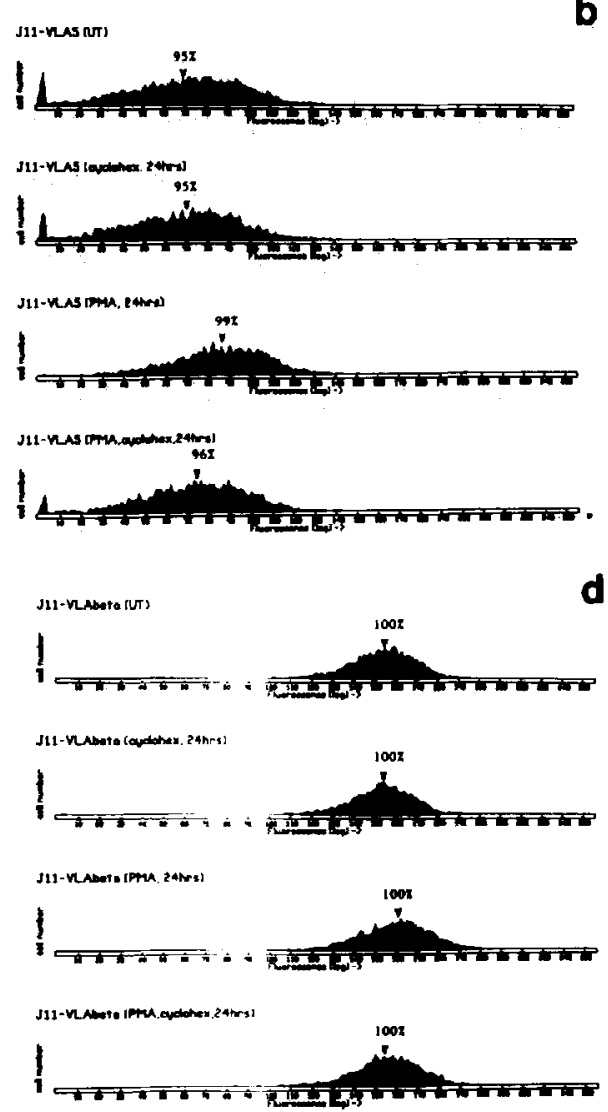

number vs, fluorescent intensity on a 3 -decade, 256 channel $\log$ scale (intensity doubles every 24 channels). The background staining with both pooled mouse IgG (control for $\alpha 4$ and $\alpha 5$ ) and pooled rat IgG (control for $\alpha 6$ and $\beta 1$ ) showed $90 \%$ of the cells in the "negative" peak seen at the extreme left in (b) and (c). The arrowheads identify the "mean" channel and the numbers above give the percentage of positive cells.

TABLE 2. Inhibition of Jurkat cell attachment to fibronectin and laminin with antibodies to $\beta 1$ integrins $^{1}$

\begin{tabular}{lccr}
\hline \multirow{2}{*}{$\begin{array}{l}\text { Monoclonal } \\
\text { antibody }\end{array}$} & Specificity & Fibronectin & Laminin \\
\hline None & & $55 \pm 3$ & $62 \pm 4$ \\
AIIB2 & $\beta 1$ & $1 \pm 2$ & $1 \pm 1$ \\
B1E5 & $\alpha 5$ & $13 \pm 4$ & $52 \pm 3$ \\
GoH3 & $\alpha 6$ & $52 \pm 6$ & $13 \pm 4$ \\
\hline
\end{tabular}

${ }^{1}$ The attachment assays were carried out as described in Materials and Methods using Jurkat cells that had been treated for 2 days with PMA. Prior to addition of colls to the Jurkat cels that had been treated for 2 days with PMA. Prior to addition of cells to the
substrate the cells were incubated for 15 minules with the various monoclonal antibodsubstrate, the cells were incubated for 15 minules with the various monoclonal antibod-
ies $(1: 10$ final dilution of hybridoma supernatants) and then transferred to the fibronecies (1:10 final dilution of hybridoma supernatants) and then transferred to the fibronec-
tin- or laminin-coated wells. Data points represent means and standard deviations based on triplicate samples in a single experiment. The experiment was repeated three times with similar results.

After 24-72 hours treatment with PMA, the $\alpha 5$ specific Mab B1E5 blocks $76 \%$ of adhesion on fibronectin, whereas the $\alpha 6$ specific Mab GoH3 blocks $80 \%$ of the attachment on laminin. In contrast, a Mab directed at the common $\beta 1$-chain, AIIB2, blocks $99 \%$ of the attachment on both substrates. Thus the $\beta 1$-integrin family mediates both the early (Chan et al., 1991) and late increases in attachment to fibronectin and laminin fol- 
TABLE 3. Effect of staurosporine on the function and expression of $\beta 1$-integrins ${ }^{\mathrm{t}}$

\begin{tabular}{|c|c|c|c|c|}
\hline \multirow[b]{2}{*}{ Treatment } & \multicolumn{2}{|c|}{ Binding activity } & \multicolumn{2}{|c|}{ Expression } \\
\hline & Laminin & Fibronectin & $\alpha 5$ & $\alpha 6$ \\
\hline 4 Hours & & & & \\
\hline Lntreated & $769(3 \%)$ & $7,977(32 \%)$ & 48 & 19 \\
\hline $50 \mathrm{mM}$ Staur. & $423(2 \%)$ & $6,807(27 \%)$ & 49 & 22 \\
\hline PMA & $3,184(13 \%)$ & $12,481(50 \%)$ & 45 & 17 \\
\hline $\begin{array}{l}\text { PMA +50 mM Staur. } \\
24 \text { hours }\end{array}$ & $186(1 \%)$ & $7,322(29 \%)$ & 51 & 16 \\
\hline Untreated & $534(2 \%)$ & $7,359(29 \%)$ & 51 & 25 \\
\hline $50 \mathrm{mM}$ Staur. & $300(1 \%)$ & $5,378(22 \%)$ & 45 & 31 \\
\hline PMA & $6,324(25 \%)$ & $12,898(52 \%)$ & 55 & 81 \\
\hline PMA $+50 \mathrm{mM}$ Stauro. & $778(3 \%)$ & $6,666(27 \%)$ & 55 & 35 \\
\hline
\end{tabular}

${ }^{1}$ Jurkat cells treated with PMA for a total of 4 or 24 hours (including the 4 hour period of the adhesion assayj. Adhesion and marker expressiun were measured as described in Materials and Methods. Binding activity is given as the mean number ( $\%$ of addedi of cells attached to the wells after washing (triplicate determination). Epitupe expreseion is given as the is given as the/man flun ancence wit isotype controls [ (linear scales;. Similar findings were obtained in three independen experiments.

TABI.E 4. Attachment of different T-lymphoblastic cell lines to fibronectin and laminin ${ }^{1}$

\begin{tabular}{lcccc}
\hline \multicolumn{5}{c}{ Percent attached } \\
\hline Substrate & Untreated & $\begin{array}{c}\text { PMA } \\
(4-8 \text { hours })\end{array}$ & $\begin{array}{c}\text { PMA } \\
(1-2 \text { days })\end{array}$ & $\begin{array}{c}\text { PMA } \\
(3-5 \text { days })\end{array}$ \\
\hline Fibronectin & & & & \\
HSR-2 & $27 \pm 6$ & $55 \pm 4$ & $48 \pm 9$ & - \\
CEM & $18+6$ & $56 \pm 5$ & $49 \pm 17$ & - \\
Molt-4 & $13: 4$ & $44 \pm 16$ & $48 \pm 5$ & - \\
HPB-ALL & $10 \pm 5$ & $47 \pm 6$ & $46+6$ & - \\
\hline Laminin & & & & $6 \pm 1$ \\
HSB-2 & $1-1$ & $3 \pm 1$ & $1 \pm 1$ & $16 \pm 4$ \\
CEM & $1 \div 1$ & $2 \pm 1$ & $7 \pm 5$ & $28 \pm 6$ \\
Molt-4 & $1 \pm 2$ & $2 \pm 1$ & $3 \pm 1$ & $20-5$ \\
HPB-ALI & $1 \pm 1$ & $7 \pm 1$ & $18 \div 3$ &
\end{tabular}

${ }^{1}$ Attachment of various T-lymphablastic lines to fibronectin and laminin under contro conditions and after treatment with PMA. The attachment assays were carried out a described in Materials and Methods. Iata points represent means and standard error taken from a large number of independent experiments. In some cases, cells were treated with PMA at the start of the adhesion assay; in other cases, pretreatment for up to 4 hours was performed. Data from these experiments have been combined under the heading: PMA, $4-8$ hours. The long-term pretreatment groups include data pooled from experiments in which cells were treated for 1-2 days or $3-5$ days.

lowing PMA treatment. VLA-5 ( $\alpha 5 / \beta 1)$ and VL $\Lambda-6(\alpha 6 /$ $\beta 1$ ) are the principle receptors for fibronectin and laminin, respectively, with lessor contributions from other members of the $\beta 1$ family.

The most likely targets for PMA in Jurkat cells, particularly at the low concentrations employed in the current study, are one or more isoforms of protein kinase $\mathrm{C}$ (pkC) (Knopf et al., 1986; Beyers et al., 1988; Nishizuka, 1988; Ono et al., 1988). PKCs are the best-documented receptors for phorbol esters in lymphoid cells (Imboden and Weiss, 1988). Serine/threonine kinases in the Erk (MAP kinase) family also respond to PMA in whole cells (Hoshi et al., 1988; Nel et al., 1991). However, these responses are most likely the result of C-kinase initiated phosphorylation cascades (reviewed in (Crews et al., 1992)). Protein-tyrosine kinases may also be substrates for pkCs (Veillette et al., 1988); however, direct activation by phorbol esters has not been described. In T cells, for example, tyrosine phosphorylation of the $\zeta$ chain of CD3 is not induced by either phorbol esters or diacylglycerol (Klausner and Samelson, 1991). Recently, direct activation of phospholipase
D (PLD) in lymphocytes by phorbol esters has been postulated (Cao et al., 1990). This conclusion rests primarily on the observation that several inhibitors of C-kinase activity fail to prevent upregulation of PLD by phorbol esters in intact cells. However, phorbol esters do not up-regulate PLD in membrane preparations as one would expect if direct binding and activation occurred. Thus C-type kinases remain the only enzymes in which activation following binding of phorbol esters has been clearly documented for the purified enzyme (Ono et al., 1989).

In lymphoid cells, activation and translocation of C-type kinases occur within minutes of initiating treatment with PMA (Ase et al., 1988; Kim et al., 1989). However, continuous treatment depletes lymphoid cells of PKC activity within hours to days. Therefore, depletion as well as activation of C-kinases may contribute to the de novo synthesis of integrins following continuous treatment with PMA. The existence of multiple PKC isoforms in the Jurkat line further complicates interpretation since their relative sentivities to PMA have not been established (Koretzky et al., 1989). These issues illustrate the complexity of the pathways involved in the response to PMA and highlight the need for further investigation.

The blockade of PMA-induced matrix adhesion by staurosporine further illustrates the role of protein $\mathrm{ki}$ nases in regulating $\beta 1$ integrin function. Jurkat cells treated with PMA and staurosporine bound to the matrix molecules; however, the twofold (fibronectin) and five- to tenfold (laminin) increases in binding following PMA treatment alone were suppressed. Staurosporine blocked both the early and late responses to PMA. Inhibition of the early response did not result from loss of receptors at the cell surface since the expression of the $\alpha 5$ and $\alpha 6$ epitopes were unchanged. In contrast, inhibition of the late increase in laminin binding activity reflects, in part, suppression of new receptor synthesis since staurosporine prevents the threefold rise in $\alpha 6$ expression after 20 hours with PMA. Thus increases in the activities of one or more protein kinases mediate both the initial increase in the avidity of $\beta 1$ integrins and the late increase in expression of functional $\alpha 6 / \beta 1$ receptors at the cell surface.

Finally, the responses of T-lymphoblastic cell lines to PMA indicate a link between developmental maturity and expression of functional receptors for fibronectin and laminin. Previous studies document phenotypic changes in T-lymphoblastic cell lines consistent with cellular differentiation and activation following treatment with PMA (Stoolman and Ebling, 1989). In the current study, binding to laminin developed rapidly (4-48 hours) in the most phenotypically mature cell lines (HPB-ALL and Jurkat). I ines showing intermediate levels of maturity, Molt-4 and CEM, required longer incubations with PMA (48-120 hours). The least mature line, HSB-2, required the longest incubation (120 hours) and showed the weakest response. Thus only T-lymphoblastic lines resembling thymocytes at or beyond stage II develop significant laminin binding activity in response to PMA. In contrast, all cell lines showed constitutive binding to fibronectin. We propose that in human T-lymphoblastic malignancies, as in their normal counterparts (Wadsworth et al., 1992), the 
expression of functional receptors for laminin is developmentally regulated.

\section{ACKNOWLEDGMENTS}

The authors thank Hazel Ebling and Patricia Perone for their expert technical assistance. This work was supported by NIH grants K08-CA00959 and R01CA49256 to L.M.S., and ACS grant IM432 to J.V.

\section{LITERATURE CITED}

Ase, K., Berry, N., Kikkawa, U., Kishimoto, A., and Nishizuka, Y. (1988) Differential down-regulation of protein kinase $\mathrm{C}$ subspecies in KM3 cells. FEBS Lett., 236:396-400.

Beyers, A.D., Hanekom, C., Rheeder, A., Strachan, A.F., Wooten, M.W., and Nel, A.E. (1988) Characterization of protein kinase C and its isoforms in human T lymphocytes. J. Immunol., 141:3463-3470.

Brown, D.L., Phillips, D.R., Damsky, C.H., and Charo, I.F. (1989) Synthesis and expression of the fibroblast fibronectin receptor in human monocytes. J. Clin. Invest., 84:366-370.

Cao, Y.Z., Reddy, C.C., and Mastro, A.M. (1990) Evidence for protein kinase $\mathrm{C}$ independent activation of phospholipase $\mathrm{D}$ by phorbol esters in lymphocytes. Biochem. Biophys. Res. Commun., 171:955962.

Cardarelli, P.M., and Pierschbacher, M.D. (1986) T-lymphocyte differentiation and the extracellular matrix: identification of a thymocyte subset that attaches specifically to fibronectin. Proc. Natl. Acad. Sci. USA, 83:2647-2651.

Cardarelli, P.M., and Pierschbacher, M.D. (1987) Identification of fibronectin receptors on T lymphocytes. J. Cell Biol, 105:499-506.

Cardarelli, P.M., Crispe, I.N., and Pierschbacher, M.D. (1988) Preferential expression of fibronectin receptors on immature thymocytes. J. Cell Biol, 106:2183-2190.

Carrel, S., Mach, J.P., Miescher, G., Salvi, S., Giuffre, L., Schreyer, M. and Isler, P. (1987) Phorbol 12-myristate 13-acetate induces surface expression of T3 on human immature cell lines with and without concomitant expression of the $T$ cell antigen receptor complex. Cur. J. Immunol., 17:1079-1087.

Chan, B.M., Wong, J.G., Rao, A., and Hemler, M.E. (1991) T cell receptor-dependent, antigen-specific stimulation of a murine $T$ cell clone induces a transient, VLA protein-mediated binding to extracellular matrix. J. Immunol., 147:398-404.

Crews, C.M., Alessandrini, A., and Erikson, R.L. (1992) Erks: Their fifteen minutes has arrived. Cell Growth Diff, $3: 135-142$

Delia, D., Greaves, M.F., Newman, R.A., Sutherland, D.R., Minowada J., Kung, P., and Goldstein, G. (1982) Modulation of T leukaemic cell phenotype with phorbol ester. Int. J. Cancer, 29:23-31.

Hall, D.E., Reichardt, L.F., Crowley, E., Holley, B., Moezzi, H., Sonnenberg, A., and Damsky, C.H. (1990) The alpha 1/beta 1 and alpha 6/beta 1 integrin heterodimers mediate cell attachment to distinct sites on laminin. J. Cell Biol., 110:2175-2184

Hemler, M.E, Huang, C., and Schwarz, L. (1987) The VLA protein family. Characterization of five distinct cell surface heterodimers each with a common 130,000 molecular weight beta subunit. J. Biol Chem 262:3300-3309.

Hoshi, M., Nishida, E., and Sakai, H. (1988) Activation of a Ca2+ inhibitable protein kinase that phosphorylates microtuble-associ ated protein 2 in vitro by growth factors, phorbol esters, and serum in quiescent cultured human fibroblasts. J. Biol. Chem., 263:53965401 .

Imboden, J.B., and Weiss, A. (1988) The initiation of human T Jymphocyte activation. Prog. Allergy., 42:246-279.

Kim, D.K., Lancki, D.W., Hui, F.H., and Fitch, F.W. (1989) Protein kinase $\mathrm{C}$-dependent and -independent mechanisms of cloned murine $\mathrm{T}$ cell proliferation. The role of protein kinase $\mathrm{C}$ translocation and protein kinase C activity. J. Immunol, 142:616-622.

Klausner, R.D., and Samelson, L.E. (1991) T cell antigen receptor activation pathways: The tyrosine kinase connection. Cell, 64:875878.

Knopf, J.L., Lee, M.H., Sultzman, I.A., Kriz, R.W., Loomis, C.R.,
Hewick, R.M., and Bell, R.M. (1986) Cloning and expression of multiple protein kinase C cDNAs. Cell, 46:491-502.

Koretzky, G.A., Wahi, M., Newton, M.E., and Weiss, A. (1989) Heterogeneity of protein kinase $\mathrm{C}$ isoenzyme gene expression in human $\mathrm{T}$ cell lines. Protein kinase C-beta is not required for several $\mathrm{T}$ cell functions. J. Immunol., 143:1692-1695.

Nel, A.E., Hanekom, C., and Hultin, L. (1991) Protein kinase C plays a role in the induction of tyrosine phosphorylation of lymphoid microtubule-associated protein-2 kinase Evidence for a CD3-associated cascade that includes pp561ck and that is defective in HPB-ALI.J. Immunol., 147P:1933-1939.

Nishizuka, Y. (1988) The molecular heterogeneity of protein kinase C and its implications for cellular regulation. Science, 334:661-665.

Ono, Y., Fujii, 'T', Ogita, K., Kikkawa, U., Igarashi, K., and Nishizuka, Y. (1988) The structure, expression, and properties of additional members of the protein kinase C family. J. Biol. Chem., 263:69276932

Ono, Y., Fujii, T., Igarashi, K., Kuno, T., Tanaka, C., Kikkawa, U., and Nishizuka, Y. (1989) Phorbol ester binding to protein kinase C requires a cysteine-rich zinc-finger-like sequence. Porc. Natl. Acad. Sci. USA, 86:4868-4871.

Sanchez-Madrid, F., De Landazuri, M.O. Morago, G., Cebrian, M. Acevedo, A., and Bernabeu, C. (1986) VI.A-3: a novel polypeptide association within the VLA molecular complex: Cell distribution and biochemical characterization. Eur. J. Immunol., 16:1343-1349.

Savagner, P., Imhof, B.A., Yamada, K.M., and Thiery, J.P. (1986) Homing of hemopoietic precursor cells to the embryonic thymus: characterization of an invasive mechanism induced by chemotactic peptides. J. Cell Biol., 103:2715-2727.

Savagner, P., Bauvois, B., Deugnier, M.A., Imhof, B.A., and Thiery, J.P. (1988) Aspects of haemopoietic cell dynamics: ontogeny and targeted migration. Ann. Inst. Pasteur. Immunol., 139:409-431.

Shimizu, Y., van Seventer, G.A., Horgan, K.J., and Shaw, S. (1990a) Costimulation of proliferative responses of resting CD4 $+\mathrm{T}$ cells by the interaction of VLA-4 and VLA-5 with fibronectin or VLA-6 with laminin. J. Immunol, 145:59-67.

Shimizu, Y, van Seventer, G.A., Horgan, K.J., and Shaw, S. (1990b) Roles of adhesion molecules in T-cell recognition: fundamental similarities between four integrins on resting human T cells (LFA-1, VLA-4, VLA-5, VLA-6) in expression, binding, and costimulation. Immunol. Rev., 114:109-143.

Shimizu, Y., van Seventer, G.A., Horgan, K.J., and Shaw, S. (1990c) Regulated expression and binding of three VLA (beta 1) integrin receptors on T cells. Nature, $345: 250-253$

Sonnenberg, A., Modderman, P.W., and Hogervorst, F. (1988) Laminin receptor on platelets is the integrin VLA-6. Nature, $3.36: 487-$ 489 .

Stoolman, L.M., and Ebling, H. (1989) Adhesion Molecules of Cultured Hematopoietic Malignancies: a calcium-dependent lectin is the principle mediator of binding to the high endothelial venule of lymph nodes. J. Clin. Invest., 84:1196-1205.

Takada, Y., Strominger, J.L., and Hemler, M.E. (1987) The very late antigen family of heterodimers is part of a superfamily of molecules involved in adhesion and embryogenesis. Proc. Natl. Acad. Sci. USA, 84:3239-3243.

Veillette, A., Horak, I.D, and Bolen, J.B. (1988) Post-translational alterations of the tyrosine kinase $\mathrm{p} 561 \mathrm{ck}$ in response to activators of protein kinase C. Oncogene. Res., 2:385-401.

Wadsworth, S. Halvorson, M.J, and Coligan, J.E. (1992) Developmentally regulated expression of the beta 4 integrin on immature mouse thymocytes. J. Immunol., 149:421-428.

Wayner, E.A., Carter, W. G., Piotrowicz, R.S, and Kunicki, T.J. (1988) The function of multiple extracellular matrix receptors in mediating cell adhesion to extracellular matrix: preparation of monoclonal antibodies to the fibronectin receptor that specifically inhibit cell adhesion to fibronectin and react with platelet glycoproteins Ic-IIa. J. Cell Biol., 107:1881-1891.

Wayner, E.A., Garcia-Pardo, A., Humphries, M.J., McDonald, J.A. and Carter, W.G. (1989) Identification and characterization of the T lymphocyte adhesion receptor for an alternative cell attachment domain (CS-1) in plasma fibronectin. J. Cell Biol., 109:1321-1330.

Wilkins, J.A., Stupack, D., Stewart, S., and Caixia, S. (1991) Beta 1 integrin-mediated lymphocyte adherence to extracellular matrix is enhanced by phorbol ester treatment. Eur. J. Immunol., 21:517-522. 